\title{
Comparative study of depth-dose distributions for beams of light and heavy nuclei in tissue-like media
}

\author{
Igor Pshenichnov* \\ Frankfurt Institute for Advanced Studies, J.-W. Goethe University, 60438 Frankfurt am Main, Germany and \\ Institute for Nuclear Research, Russian Academy of Science, 117312 Moscow, Russia \\ Igor Mishustin \\ Frankfurt Institute for Advanced Studies, J.-W. Goethe University, 60438 Frankfurt am Main, Germany and \\ Kurchatov Institute, Russian Research Center, 123182 Moscow, Russia \\ Walter Greiner \\ Frankfurt Institute for Advanced Studies, J.-W. Goethe University, 60438 Frankfurt am Main, Germany
}

(Dated: February 6, 2020)

\begin{abstract}
We study the energy deposition by light and heavy nuclei in tissue-like media as used for cancer therapy. The depth-dose distributions for protons, ${ }^{3} \mathrm{He},{ }^{12} \mathrm{C},{ }^{20} \mathrm{Ne}$, and ${ }^{58} \mathrm{Ni}$ nuclei are calculated within a Monte Carlo model based on the GEANT4 toolkit. These distributions are compared with each other and with available experimental data. It is demonstrated that nuclear fragmentation reactions essentially reduce the peak-to-plateau ratio of the dose profiles for deeply penetrating energetic ions heavier than ${ }^{3} \mathrm{He}$. On the other hand, all projectiles up to ${ }^{20} \mathrm{Ne}$ were found equally suitable for therapeutic use at low penetration depths.

PACS numbers: $87.53 .-\mathrm{j}$, 87.53.Pb, 87.53.Wz
\end{abstract}

Proton and ion beams of intermediate energies are widely used nowadays for cancer treatment [1]. Irradiation of deeply-seated tumors without destroying healthy tissues becomes possible because such heavy projectiles deliver enhanced dose at the very end of their range in tissues, close to the Bragg peak. In the first pioneering studies, see Ref. 2] for a historical review, the choice of projectile nuclei and beam energy for radiation therapy was mainly determined by the parameters of accelerators available in physics laboratories. Several experimental studies with homogeneous phantoms were also performed. Accurate measurements of the depth-dose distributions for ${ }^{12} \mathrm{C},{ }^{18} \mathrm{O}$ and ${ }^{20} \mathrm{Ne}$ ions in water were made at GSI, Germany and at NIRS, Japan [3].

It is expected [1] that carbon ions are more advantageous in radiotherapy compared to protons because of (1) reduced longitudinal and lateral scattering in tissues, (2) increased relative biological effectiveness (RBE) close to the Bragg peak, and (3) the possibility to monitor the beam range by the positron emission tomography (PET). The latter is realized via the detection of positron emission by nuclear fragments (e.g. ${ }^{10} \mathrm{C}$ and ${ }^{11} \mathrm{C}$ ) created in fragmentation of beam nuclei. Currently GSI is using ${ }^{12} \mathrm{C}$ beams for treatment and continues research with such beams in different phantoms [4]. Two new dedicated hospitals in Heidelberg, Germany, and in Pavia, Italy, will soon provide cancer treatments with carbon and proton beams [1]. Two hospital-based facilities in Japan, HI-

\footnotetext{
*Corresponding author: pshenich@fias.uni-frankfurt.de
}

MAC at Chiba and HIBMC at Hyogo are using carbon beams for cancer treatment too [1]. The feasibility of ${ }^{3} \mathrm{He}$ beams for therapy is currently under investigation [5].

The aim of this paper is to provide theoretical guidance in choosing the ion kind and beam energy from the view point of their suitability for cancer therapy. This is done by comparison of depth-dose distributions for various projectiles, from protons to nickel ions at different energies. We take into account electromagnetic and hadronic interactions of primary and secondary particles. We pay special attention to nuclear fragmentation reactions which reduce the fluence of primary beam ions. We believe that the lack of systematic experimental studies with ions other than carbon can be partially filled with calculations.

The propagation of protons and nuclei in tissue-like media is studied with a Monte Carlo Model for Heavyion Therapy (MCHIT) based on the GEANT4 toolkit 6 , 7] (version 8.2). Here we briefly describe the choice of models employed in our calculations, as more details can be found in Refs. [8, 9].

The energy loss of primary and secondary charged particles due to electromagnetic interactions is calculated with a set of Monte Carlo models called 'standard electromagnetic physics'. This accounts for energy loss and straggling of charged particles due to interaction with atomic electrons as well as multiple Coulomb scattering on atomic nuclei. At each simulation step, the ionisation energy loss of a charged particle is calculated according to the Bethe-Bloch formula. There the mean excitation potential of water molecules was set to $77 \mathrm{eV}$, i.e. to the value which better describes the set of available 
data on depth-dose distributions measured with carbonion beams $[3,4]$.

The binary cascade model [6, 7] is used to describe the collisions of energetic nucleons and ions with protons and nuclei of the medium. After the cascade stage of interaction the decay of excited nuclear remnants is considered by employing several models. The WeisskopfEwing model is used for the description of evaporation of nucleons from nuclei at excitation energies below 3 $\mathrm{MeV}$ per nucleon. The Statistical Multifragmentation Model (SMM) [10] is used to describe multi-fragment break-up of highly-excited residual nuclei at excitation energies above $3 \mathrm{MeV}$ per nucleon. The SMM includes as its part the Fermi break-up model for describing an explosive disintegration of highly-excited light nuclei with $Z \leq 8$ and $A \leq 18$.

Below we present some results obtained with the MCHIT model. Figure 1 shows 3D dose distributions for the proton and ${ }^{12} \mathrm{C}$ beams with Gaussian intensity profiles of $4 \mathrm{~mm}$ FWHM propagating through polymethylmethacrylate (PMMA). The dose is defined as the deposited energy per unit volume per beam projectile and measured in $\mathrm{MeV}$ per $\mathrm{mm}^{3}$. The beam energies are chosen so that the stopping point of both projectiles is located at the same distance of $\sim 170 \mathrm{~mm}$, corresponding to the Bragg peak in linear energy deposition. Secondary nucleons and nuclear fragments are produced at different depths and have broad angular and energy distributions. Their effect must be carefully evaluated, especially in the regions beyond the Bragg peak. One can clearly see that multiple scattering of beam particles leads to radial widening of the dose field. This effect is considerably larger for protons than for carbon ions. The MCHIT model provides the possibility for accurate calculations of the spatial dose distributions as needed for cancer therapy.

As carbon-ion beams are currently widely used in heavy-ion therapy, it is important to know well the evolution of their depth-dose profiles with increasing beam energy. Corresponding distributions for 200 and $400 \mathrm{~A}$ $\mathrm{MeV}{ }^{12} \mathrm{C}$ nuclei calculated with the MCHIT model are shown in Fig. 2(a) and compared to the experimental data [4]. Results for 600,800 and $1000 \mathrm{~A} \mathrm{MeV}{ }^{12} \mathrm{C} \mathrm{nu}-$ clei are shown in Fig. 2(b). The depth-dose distributions were obtained in terms of the average linear energy deposition per beam particle and expressed in $\mathrm{MeV} / \mathrm{mm}$. They were calculated by splitting a cubic phantom into thin slices and calculating the energy deposited in each of the slices. In calculations the beam energy spread was assumed to be Gaussian with FWHM of $0.2 \%$

Secondary nucleons and nuclei can be created in ${ }^{12} \mathrm{C}$ projectile fragmentation reactions at any point within the projectile range $R_{p}$, before the Bragg peak. The range of a secondary fragment $R_{A, Z}$ with mass $A$ and charge $Z$ with the energy per nucleon $E$ equal to that of the projectile with mass $A_{p}$ and charge $Z_{p}$ can be expressed
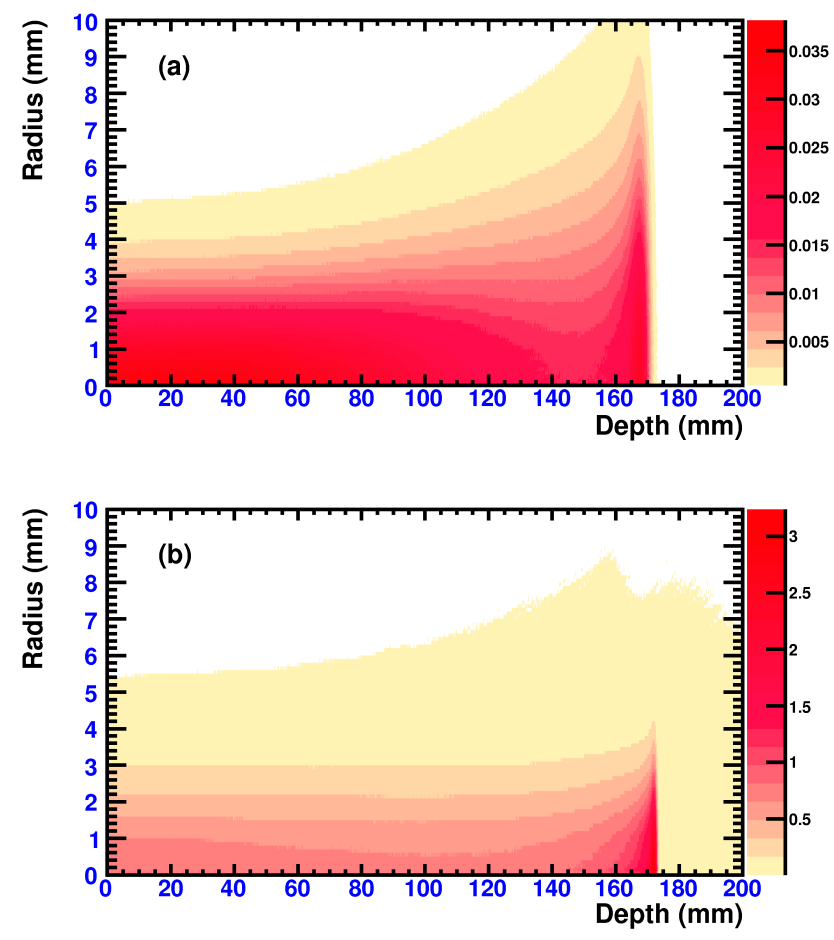

FIG. 1: Color online. MCHIT simulation of volume dose distributions, in $\mathrm{MeV} / \mathrm{mm}^{3}$ per beam particle, for $4 \mathrm{~mm}$ FWHM beams of (a) $170 \mathrm{MeV}$ protons and (b) $330 \mathrm{~A} \mathrm{MeV}{ }^{12} \mathrm{C}$ ions in PMMA. Notice the dose scales are different in two panels.

as $R_{A, Z}(E)=\frac{A}{Z^{2}} \frac{Z_{p}^{2}}{A_{p}} R_{p}(E)$, see e.g. Ref. [5]. Depending on the mass and charge of produced fragments they either propagate beyond the Bragg peak, like e.g. protons, neutrons deutrons or helium nuclei, or stop before the distal edge of the peak, like e.g. ${ }^{10} \mathrm{C}$ or ${ }^{11} \mathrm{C}$ projectile fragments. These properties of nuclear fragments were already demonstrated by the calculations for carbon-ion beams within the MCHIT model [8, 9]. We have found that also the recent data [4] are well described by the MCHIT model, including the contribution of secondary particles beyond the Bragg peak, as shown in Fig. 2(a).

As one can see from Fig. 2, the fragmentation of projectile nuclei reduces the fluence of primary beam nuclei progressively with increasing beam energy. As found in Ref. [4], at $400 \mathrm{~A} \mathrm{MeV}$ beam energy about $70 \%$ of primary ${ }^{12} \mathrm{C}$ ions change their charge due to nuclear fragmentation reactions. Fortunately, this does not deteriorate too much the suitability of such beams for heavy-ion therapy. As seen in Fig. 2(a), the peak-to-entrance ratio is still around 4, as compared to 6.5 at $200 \mathrm{~A} \mathrm{MeV}$.

The carbon ions with energies above $500 \mathrm{~A} \mathrm{MeV}$ are not suitable for therapeutic use, as their ranges in human tissues extend beyond typical dimensions of a patients' body. As follows from Fig. 2(b), at high energies the calculations predict a very large contribution of frag- 

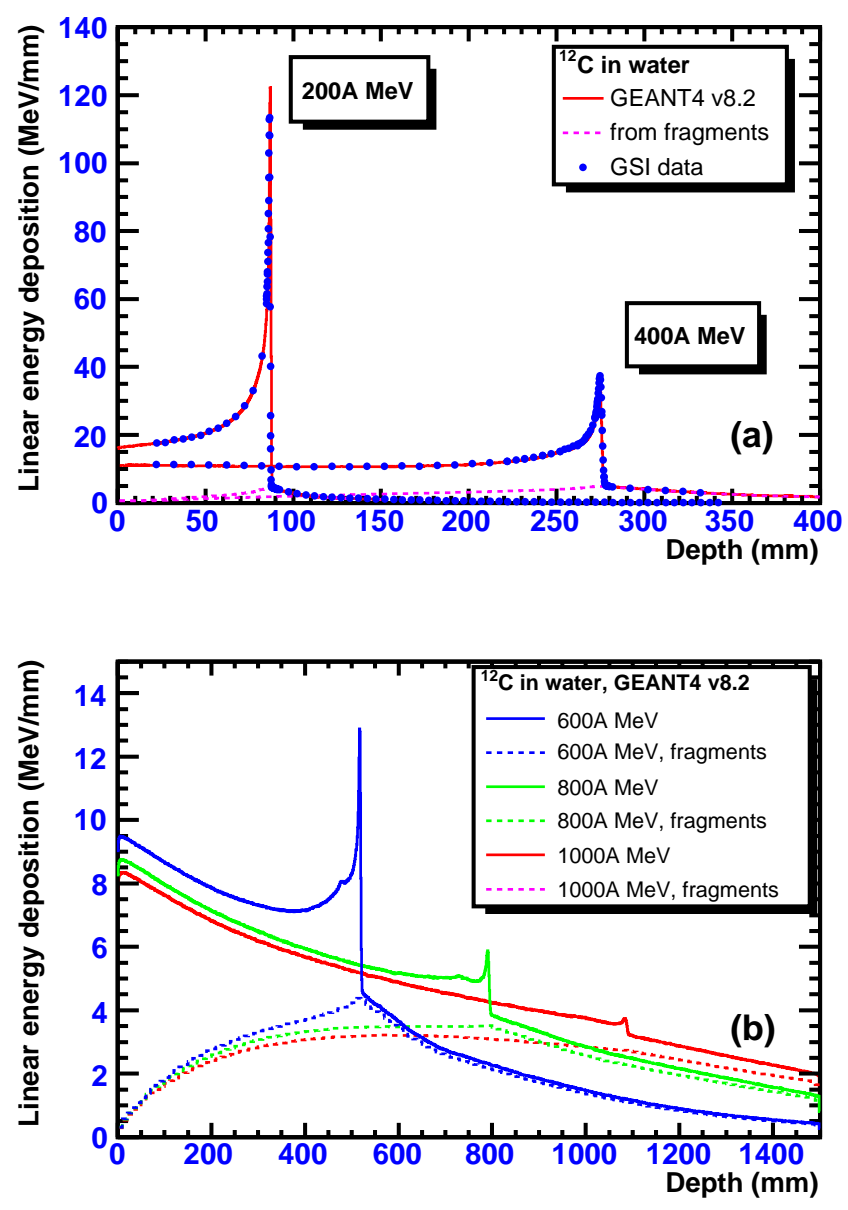

FIG. 2: Color online. Calculated depth-dose distributions for (a) 200 and $400 \mathrm{~A} \mathrm{MeV}$ and (b) 600, 800 and $1000 \mathrm{~A}$ $\mathrm{MeV}{ }^{12} \mathrm{C}$ beams in water (solid histograms). Contributions from all charged fragments with $Z \neq 6$ are shown by dashed histograms. Corresponding experimental data [4] are shown by points.

mentation reactions leading to the decreasing height and disappearance of the Bragg peak. This makes the peakto-entrance dose ratio unacceptably small. As a result, the contribution from secondary nuclear fragments beyond the Bragg peak is very large.

This also demonstrates inefficiency of passive beam delivery systems in heavy-ion therapy. In this technique, the beam energy is adjusted by inserting specially designed ridge filters, range shifters and compensators (boluses) in front of a patient. In calculations such beam modulating elements can be roughly represented by additional water thickness. Secondary particles produced in beam fragmentation on such elements will be responsible for essential unwanted dose deposition beyond the Bragg peak.

The depth-dose distributions for various ion species and beam energies are shown in Fig. 3 for two preselected positions of the Bragg peak at 60 and $360 \mathrm{~mm}$, respectively. These distances represent two extreme cases of proton and heavy-ion therapy corresponding to shallow and deep sitting tumors. The beam energies for protons, ${ }^{3} \mathrm{H},{ }^{12} \mathrm{C},{ }^{20} \mathrm{Ne}$ and ${ }^{58} \mathrm{Ne}$ ions were chosen different in such a way that these projectiles have similar ranges in water, $\sim 60 \mathrm{~mm}$ or $\sim 360 \mathrm{~mm}$. In Fig. 3(a) the calculated distributions for all projectiles, but ${ }^{12} \mathrm{C}$, were multiplied by the corresponding factors rescaling their entrance doses to the level of ${ }^{12} \mathrm{C}$ ions. This facilitates the comparison of the depth-dose distributions for different beams. Although the same factors were also applied in Fig. 3(b), this rescaling yields the same entrance doses only for protons and ${ }^{3} \mathrm{He}$. For ${ }^{20} \mathrm{Ne}$ and ${ }^{58} \mathrm{Ni}$ projectiles the rescaled entrance dose is $\sim 20 \%$ higher than for ${ }^{12} \mathrm{C}$.

Experimental data for ${ }^{58} \mathrm{Ni}$ and ${ }^{20} \mathrm{Ne}$ beams are also presented in Fig. 3 for comparison. One can see that the agreement between the theory and the experiments is generally quite good. We have tried to eliminate small discrepancies by fine tuning the beam energy for ${ }^{58} \mathrm{Ni}$ and ${ }^{20} \mathrm{Ne}$ within experimental uncertainties. We have found that calculations with $397 \mathrm{~A} \mathrm{MeV}{ }^{58} \mathrm{Ni}$ beam energy can well describe the data of Ref. [11] where 400 A $\mathrm{MeV}$ beam energy was quoted. Also, the Bragg peak position reported in Ref. [3] for $670 \mathrm{~A} \mathrm{MeV}{ }^{20} \mathrm{Ne}$ beam is better reproduced by calculations with beam energy of $665 \mathrm{~A} \mathrm{MeV}$. However, a noticeable discrepancy in shape between the MCHIT results and the data remains for ${ }^{20} \mathrm{Ne}$ ions, as seen in Fig. 3(b).

Results presented in Fig. 3(a) show that all beams from proton to Ni have a similar shape of the depth-dose distribution. In this situation the suitability of a specific beam for the cancer treatment is dictated by its biological effects. In particular, the ${ }^{58} \mathrm{Ni}$ beam is probably not acceptable because of the very high entrance dose (about $250 \mathrm{MeV} / \mathrm{mm}$ ), which will destroy the healthy tissues. By inspecting Fig. 3(b) we conclude that $665 \mathrm{~A} \mathrm{MeV}^{20} \mathrm{Ne}$ and $1346 \mathrm{~A} \mathrm{MeV}{ }^{58} \mathrm{Ni}$ beams are less suitable for cancer therapy compared to lighter projectiles due to increased beam fragmentation. Indeed, the peak value for ${ }^{20} \mathrm{Ne}$ is only twice as large as the entrance dose. For ${ }^{58} \mathrm{Ni}$ the situation is even worse: the Bragg peak is very weak and the entrance dose is higher than the Bragg peak. This shows clear advantage of lighter projectiles like protons, ${ }^{3} \mathrm{He}$ and ${ }^{12} \mathrm{C}$ ions for treatment of deep-seated tumors.

In summary, our systematic study of depth-dose distributions of nuclear beams in tissue-like medium shows that the shape of the energy deposition profile depends rather on the ion range than on the ion mass. At relatively low beam energies corresponding to small penetration depths, nuclear fragmentation reactions do not play a significant role, so that the Bragg peak is well pronounced, and the tail due to secondary particles is small for all considered beams. In this case the depth-dose distributions for various nuclei are similar to each other, and not very different from the distribution calculated for 

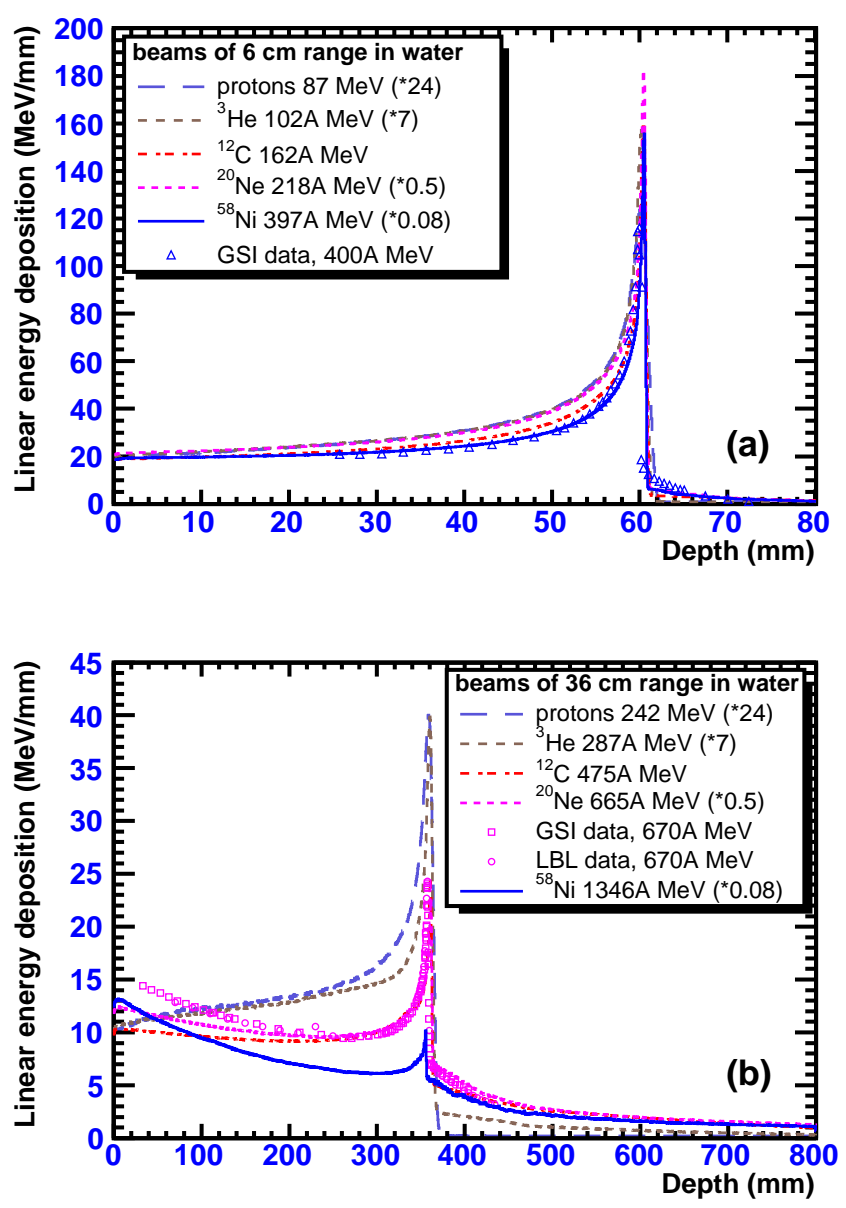

FIG. 3: Color online. Calculated depth-dose distributions for (a) beams of protons $(87 \mathrm{MeV}),{ }^{3} \mathrm{He}(102 \mathrm{~A} \mathrm{MeV}),{ }^{12} \mathrm{C}$ $(162 \mathrm{~A} \mathrm{MeV}),{ }^{20} \mathrm{Ne}(218 \mathrm{~A} \mathrm{MeV})$ and ${ }^{58} \mathrm{Ni}(397 \mathrm{~A} \mathrm{MeV})$ nuclei and (b) for protons $(242 \mathrm{MeV}),{ }^{3} \mathrm{He}(287 \mathrm{~A} \mathrm{MeV}),{ }^{12} \mathrm{C}(475 \mathrm{~A}$ $\mathrm{MeV}),{ }^{20} \mathrm{Ne}(665 \mathrm{~A} \mathrm{MeV})$ and ${ }^{58} \mathrm{Ni}(1346 \mathrm{~A} \mathrm{MeV})$ nuclei in water (histograms). The distributions for all, but ${ }^{12} \mathrm{C}$ nuclei, were multiplied by the rescaling factors (indicated in the legend) which normalize their entrance dose to the one for ${ }^{12} \mathrm{C}$ beam. Experimental data for ${ }^{58} \mathrm{Ni}$ ions at $400 \mathrm{~A} \mathrm{MeV} \mathrm{[11]} \mathrm{and}$ for ${ }^{20} \mathrm{Ne}$ ions at $670 \mathrm{~A} \mathrm{MeV} \mathrm{[3]}$ are shown by various symbols.

carbon ions, which is currently the only modality used in ion therapy. The peak-to-entrance dose ratio is large for all beam nuclei, from protons to nickel nuclei.

The depth-dose distributions for various nuclei are very different at high beam energies, i.e. for deeply penetrating beams. In this case one can benefit from using protons or ${ }^{3} \mathrm{He}$ ions, with their largest peak-to-entrance dose ratio and minimal contribution from nuclear fragmentation. However, the Bragg peak for protons is less sharp compared to more heavy projectiles due to the longitu- dinal and lateral spread-out of the beam particles associated with the multiple scattering processes. We found that ${ }^{3} \mathrm{He}$ beams can provide a reasonable alternative to ${ }^{12} \mathrm{C}$ beams in the case of large penetration depths, if the lateral beam scattering is not very crucial. One needs experimental data on the depth-dose distributions of ${ }^{3} \mathrm{He}$ beams to test the validity of the MCHIT model for these ions.

Here we have considered only distributions of physical doses from beams of various nuclei. The proper assessment of suitability of a certain nuclear beam for cancer therapy should also include its specific biological effects. As mentioned by several authors [1, 2], ions which are much heavier than carbon nuclei have very high linear energy transfer even at the entrance point at patients' body that makes difficult to save healthy tissues located at the beam path. As follows from our calculations, similar deficiency is observed already in the distributions of the physical doses for nuclei heavier than ${ }^{20} \mathrm{Ne}$. Taking into account these observations we conclude that protons, ${ }^{3} \mathrm{He},{ }^{12} \mathrm{C}$ and, possibly, ${ }^{16} \mathrm{O}$ are the most suitable beams to be used in future ion therapy facilities.

This work was partly supported by Siemens Medical Solutions. We are grateful to Prof. Hermann Requardt for the discussions which stimulated the present study. We are indebted to Dr. Dieter Schardt for useful discussions and for providing us the data tables for the depthdose distributions of carbon ions.

[1] U. Amaldi and G. Kraft, Rep. Prog. Phys. 68 (2005) 1861

[2] G. T. Y. Chen, J. R. Castro, and J. M. Quivey, Ann. Rev. Biohys. Bioeng. 10 (1981) 499

[3] L. Sihver, D. Schardt, and T. Kanai, Jpn. J. Med. Phys. 18 (1998) 1

[4] E. Haettner, H. Iwase, and D. Schardt, Rad. Prot. Dosim. Advance Access published on December 4, 2006; doi:10.1093/rpd/ncl402

[5] F. Fiedler, P. Crespo, and K. Parodi et al., IEEE Trans. Nucl. Sci. 53 (2006) 2252

[6] S. Agostinelli et al., (GEANT4 Collaboration) Nucl. Instrum. Meth. A 506 (2003) 250

[7] J. Allison et al., (GEANT4 Collaboration) IEEE Trans. Nucl. Sci. 53 (2006) 270

[8] I. Pshenichnov, I. Mishustin, and W. Greiner, Phys. Med. Biol. 50 (2005) 5493

[9] I. Pshenichnov, I. Mishustin, and W. Greiner, Phys. Med. Biol. 51 (2006) 6099

[10] J. P. Bondorf, A. S. Botvina, A. S. Iljinov, I. N. Mishustin, and K. Sneppen, Phys. Rept. 257 (1995) 133

[11] D. Schardt, I. Schall, H. Geissel et al., Adv. Space Res. 17 (1996) 287 